\title{
Dyaden, Gruppen und Teams: Die Rahmungen von Coachings und Supervisionen
}

\author{
Stefan Kühl
}

Zusammenfassung: In diesem Artikel wird untersucht, wie sich drei unterschiedliche Rahmungen von Beratungsgesprächen - Dyaden, Gruppen und Teams - auf die Beratungssituation auswirken.

Intuitiv ist es Beratern klar, dass es einen Unterschied macht, ob ein Klient oder eine Klientin in einem Gespräch ,unter vier Augen“, im Rahmen einer Gruppe von sich gerade erst kennenlernenden Personen oder in einem regelmäßig zusammenarbeitenden Team beraten wird. Aber was sind genau die Mechanismen die wirken? Und wie sind genau deren Auswirkungen? Der Artikel bietet eine erste Annäherung.

Schlüsselwörter: Dyade $\cdot$ Gruppe $\cdot$ Team $\cdot$ Coaching $\cdot$ Supervision

\begin{abstract}
This article examines what impact three different framings of personal oriented consulting interactions - dyads, groups and teams - have on the consulting situation. Intuitively consultants are aware that it makes a difference if a client is consulted in a face-to-face-interaction "under four eyes", in the framework of a group which just started to know each other or in a team which is working together on regular basis. However, what are exactly the mechanisms which are working in the three different settings? And what exactly are their impacts on the consulting situation? This article provides first interpretations.
\end{abstract}

Keywords: Dyad · Group · Team · Coaching $\cdot$ Supervision

Dieser Text ist als Vorüberlegung zu meinem Buch Coaching und Supervision. Personenorientierte Beratung in Organisationen. Wiesbaden. VS Verlag (Kühl, 2008) entstanden. Er diente als Vorlage für einen Vortrag auf der Jahreskonferenz 2007 des Deutschen Arbeitskreises für Gruppenpsychotherapie und Gruppendynamik. Besonders Dankwart Mattke hat sich die Mühe gemacht diesen Text zu kommentieren und mich auf einige Versäumnisse besonders in dem Teil über Gruppen hinzuweisen.

Prof. Dr. S. Kühl $(\square])$

Fakultät für Soziologie, Universität Bielefeld, D-33501 100131 Bielefeld, Deutschland

E-Mail: stefan.kuehl@uni-bielefeld.de 
Die Leistungserbringung bei der Coachings und Supervisionen - also bei personenorientierten Beratungen in Organisationen - findet in so genannten Face-to-Face-Interaktionen zwischen Beratern und Klienten statt. Unter Face-to-Face-Interaktion versteht man in der Soziologie eine verbale oder auch nonverbale Kommunikation, die unter Anwesenden stattfindet. Dazu zählen beispielsweise die Party, der gemeinsame Kneipenabend, das romantische Dinner bei Kerzenschein, die Schulstunde, die Pokerrunden, die Konsultation durch einen Arzt, Beichten und Gottesdienste, die Diskussion in einer Parlamentssitzung, die Gremiensitzungen einer Universität, Parteitage und Synoden, Gerichtsverhandlungen oder eben auch Coaching- und Supervisionsstunden.

Mit ihrer Leistungserbringung in Form von Face-to-Face-Interaktionen haben Coaching und Supervision eine Gemeinsamkeit mit den klassischen Professionen der Ärzte, Geistlichen, Theologen, Anwälte oder Lehrer. Auch Ärzte erbringen einen Großteil ihrer Leistungen im Kontakt mit ihren Patienten - durch das Abhören des Herzens, das Verbinden von Wunden oder die „längeren Beratungsgespräche (auch telefonisch)“. Rechtsanwälte erarbeiten die Fälle vorwiegend in direkter Interaktion mit ihren Klienten. Und auch die Arbeit von Geistlichen besteht aus einer Vielzahl von unterschiedlichen Face-toFace-Interaktionen: der Beichte, dem gemeinsamen Gebet, dem Gottesdienst oder dem seelsorgerischen Gespräch (siehe dazu auch Stichweh, 1996, S. 63 f.).

Die auf direkter gegenseitiger Wahrnehmung basierende Face-to-Face-Interaktion bringt den „Vorteil unmittelbarer Erlebnisverarbeitung“ mit sich. Man nimmt Informationen zeitnah über die visuellen, auditiven, geruchlichen und manchmal auch geschmacklichen und haptischen Sinne wahr. Dadurch kann man in sehr kurzer Zeit eine Vielzahl von komplexen Informationen verarbeiten. Man macht sich in wenigen Sekunden einen Eindruck, bei dem - im Vergleich beispielsweise zur Lektüre eines Buches oder zum Anschauen eines Filmes - verschiedene Informationen, unter anderem auch im Hinblick auf ihre Passung, verarbeitet werden (vgl. Luhmann, 1972, S. 248).

Dass die Leistungen in Face-to-Face-Interaktionen erbracht werden, heißt nicht, dass die Arbeit der „Interaktionsspezialisten“ ausschließlich aus der Interaktion mit Klienten besteht. Genauso wie der Arzt allein Röntgenbilder studiert oder sich in einer Ärztezeitschrift über neue Behandlungsmethoden einer Krankheit informiert und der Anwalt Gesetzestexte studiert oder Schriftsätze aufsetzt, gibt es auch bei Coaches und Supervisoren Arbeitsphasen, in denen kein Face-to-Face-Kontakt mit Klienten besteht. Sie machen sich nach Gesprächen Notizen, lesen für sie relevante wissenschaftliche Grundlagentexte oder schreiben - nicht zu vernachlässigen - Rechnungen. Aber alle diese interaktionsfreien oder interaktionsarmen Tätigkeiten stehen immer in Bezug zur eigentlichen Leistungserbringung, die in der Face-to-Face-Interaktion stattfindet.

Die Leistungserbringung über Interaktionen zieht eine Vielzahl von Konsequenzen nach sich (vgl. Luhmann, 1972, S. 248). So kann in solchen Interaktionen immer nur ein Thema zur gleichen Zeit behandelt werden. Zwar kann man gerade in geselligen Interaktionen Themen wechseln, aber die Basierung auf gegenseitiger Wahrnehmung verlangt, dass immer nur ein Thema im Zentrum einer Interaktion steht. Eine weitere Begrenzung besteht darin, dass in Interaktionen immer nur eine Person sprechen kann. Man kann den Sprecher ersetzen, aber immer nur durch einen anderen. Wenn mehrere gleichzeitig das Wort übernehmen, kommt es zu einer Krise im System, und es setzen Regulierungs- 
prozesse ein (vgl. frühe Beobachtungen bei Luhmann, 1975a, S.10 f.; Luhmann, 1975b, S. 1 ff.; siehe ausführlich Kieserling, 1999, S. 179 ff.).

Für die Untersuchung von Supervision und Coaching ist es interessant, dass die Beratungsgespräche unterschiedlich strukturiert sind. Personenorientierte Beratung in Organisationen findet statt entweder im Rahmen einer Interaktion zwischen zwei Personen, dem Berater und dem Beratenen; in Gruppen, die sich nur für Beratungen selbst treffen, oder in Teams, die sich im Rahmen ihrer regelmäßigen Zusammenarbeit coachen oder supervidieren lassen.

Ziel dieses Artikels ist zu untersuchen, wie sich diese unterschiedlichen Rahmungen in Dyaden, Gruppen oder Teams auf die Beratungssituation auswirken. Intuitiv ist es Beratern klar, dass es einen Unterschied macht, ob ein Klient oder eine Klientin in einem Gespräch „unter vier Augen“, im Rahmen einer Gruppe von sich gerade erst kennenlernenden Personen oder in einem regelmäßig zusammenarbeitenden Team beraten wird. Aber was sind genau die Mechanismen die wirken? Und wie sind genau deren Auswirkungen?

\section{Unter vier Augen: Einzelcoaching und Einzelsupervision in Dyaden}

Die wohl nach wie vor am häufigsten vorzufindende Form der personenorientierten Beratung ist die Supervision oder das Coaching „unter vier Augen“. In der Praktikerliteratur wird diese Form der Beratung als Einzelsupervision oder Einzelcoaching bezeichnet. Dieses Beratungsformat wird häufig als ,ursprüngliche Form“ der personenorientierten Beratung in Organisationen angesehen (vgl. beispielsweise Belardi, 2002, S. 85 f.). Sie spielte besonders in der Frühphase der Supervision als bevorzugte Form der Ausbildung von Sozialarbeitern und später auch Geistlichen, Lehrern, Ärzten oder Therapeuten eine wichtige Rolle. Beim Coaching von Führungskräften wird häufig ebenfalls auf die Beratung ,unter vier Augen“ zurückgegriffen, weil dabei die Vertraulichkeit der Beratung besonders gut gesichert werden kann (vgl. früh schon Looss, 1991, der eine deutliche Präferenz für diese Rahmung zu haben scheint). Auch in der Ausbildung und Weiterbildung von Supervisoren und Coaches selbst wird dieses Format häufig gewählt. Dabei trägt ein Ausbildungskandidat seinem Lehrsupervisor oder Lehrcoach eigene Fälle vor und reflektiert über seine Interventionen und Reaktionen (Schreyögg, 1992, S. 13).

Wenn man den soziologisch korrekten Begriff für diese Form von Beratungen nutzen möchte, dann würde man von „dyadischen Beratungen“ sprechen. Eine Dyade bezeichnet eine auf regelmäßige Face-to-Face-Interaktionen basierende Beziehung zwischen zwei Personen. Dyaden kennen wir besonders von Liebesbeziehungen, und man liegt vermutlich nicht völlig falsch, wenn man vieles, was in einer Liebesbeziehungen abläuft, dadurch erklärt, dass lediglich zwei Personen an einer solchen Beziehung beteiligt sind (vgl. beispielsweise Kaufmann 2004 über die Interaktionsschwierigkeiten am „Morgen danach"). Dyaden können sich jedoch auch in Organisationen - zum Beispiel beim Zusammenspiel zweier Geschäftspartnerinnen oder bei so genannten Doppelspitzen in der Politik - ausbilden. Eine wichtige Rolle spielen Dyaden aber besonders in regelmäBigen Interaktionen zwischen Dienstleistern und Klienten - im monatlichen Gespräch zwischen Arzt und Patient, bei der regelmäßigen Interaktion zwischen Geistlichen und 
Beichtenden oder im Kontakt in einer Einzelberatung (vgl. die Sammlungen bei Wiese \& Becker, 1932, S. 508 ff.; Becker \& Useem, 1942, S. 13 ff.).

\section{Die Außergewöhnlichkeit von Dyaden}

Die Besonderheit von Dyaden versteht man nur, wenn man sich klar macht, dass normalerweise fast alles, was in der modernen Gesellschaft passiert, unter der Beobachtung von Dritten stattfindet. Das gilt ganz besonders auch für Organisationen. Der "Show Down“ zwischen zwei Abteilungsleitern findet in der Regel vor Publikum statt. Die Auseinandersetzungen zwischen Vorgesetzten und Untergebenen mögen hinter verschlossenen Türen ablaufen, aber nicht selten finden dann durch die Milchglasscheibe oder durch die nach außen dringenden verbalen Kampfgeräusche auch diese Auseinandersetzungen ihre Beobachter. Selbst an Gesprächen mit wichtigen Kunden sind häufig auch weitere Organisationsmitglieder beteiligt - und sei es nur in Form des still zuhörenden Vorgesétzten. Dass es diese Dritten gibt, hat enorme disziplinarische Wirkungen für alle Beteiligten - gerade auch für den hierarchisch Höherstehenden (so schon sehr früh Simmel, 1992, S. 124 ff.; siehe allgemein auch Kaube, 2004).

Erst vor dem Hintergrund der Omnipräszenz von Dritten in der Gesellschaft wird die Besonderheit von Dyaden wie Liebesbeziehungen zwischen zwei Personen oder Beziehungen alleinerziehender Mütter zum einzigen Kinddeutlich. In der Regel bilden sich in Gesellschaften Mechanismen aus, die den kompletten Rückzug von Personen in dyadische Beziehungen zu unterbinden suchen. Jugendbands und Freundeskreise entwickeln Mechanismen, mit denen sie versuchen zu verhindern, dass sich zwei Mitglieder ihrer Gruppe durch eine intensive dyadische Liebesbeziehung entziehen. Von vielen kommunistischen und religiösen Gemeinschaften wissen wir, dass sie eine zu starke Intimisierung in Dyaden sanktionieren und beispielsweise Promiskuität oder häufigen Partnerwechsel fördern, um dies zu verhindern. Dieser Verhinderungsmechanismus lässt sich selbst bei Ehen beobachten: Mit der Einbeziehung der Familie der Ehepartner bei Hochzeitszeremonien, mit der zeitlichen Befristung exklusiver ehelicher Intimitäten in den Flitterwochen oder den außerehelichen Kontaktverpflichtungen, die mit einer Familienbildung verbunden sind, entstehen vielfältige Mechanismen, mit denen der Rückzug in einen dyadischen Kokon verhindert wird (vgl. Slater, 1963, S. 349 ff.).

Umso interessanter sind also Situationen in der modernen Gesellschaft, in denen wir es mit einer Dyade zu tun haben.

Das Management von Vertraulichkeit in dyadischen Beratungen

Bei der Beratung „unter vier Augen“" wird die dyadische Rahmung der Beratung durch verschiedene Maßnahmen gegenüber Interventionen Dritter abgesichert: das Festlegen von Treffen in gesonderten Räumen (teilweise außerhalb der Organisation des Klienten), das Ausschalten von Handys während der Beratung oder das Einräumen von geschützten Zeitfenstern.

Über diesen kontrollierten Ausschluss von Dritten können Dyaden eine besondere Form von Vertraulichkeit herstellen. Weil lediglich zwei Personen an einer Dyade beteiligt sind, unterliegen die in Gesprächen vermittelten Inhalte einer besonderen Form von 
Kontrolle. Diese wird nicht vorrangig durch die Selbstverpflichtung zur Vertraulichkeit hergestellt. Diese könnte ja auch in größeren Gruppen eingefordert werden. Vielmehr geht die Vertraulichkeit auf die besonderen Formen der sozialen Kontrolle von Informationen in Zweierbeziehungen zurück. Weil immer nur eine andere Person an dem Gespräch beteiligt ist, kann eine eindeutige Zurechnung stattfinden, wenn Informationen aus dem Gespräch nach außen dringen. Da beide über diese Möglichkeit wissen, kommt es in der Dyade zu einer besonderen Sicherheit bei der Wahrung von Vertraulichkeit (vgl. Simmel, 1992, S. 101).

Die Vertraulichkeit ermöglicht das Ansprechen von Themen, deren Behandlung ansonsten gerade im organisatorischen Kontext nur selten riskiert wird. Man kann ,unter vier Augen" Konflikte ansprechen, von denen andere nichts wissen sollen, Regelverstöße diskutieren, die bei Bekanntwerden bis zur Kündigung führen könnten, oder Zweifel äußern, die man normalerweise verborgen hält, weil sonst an der Loyalität gegenüber der Organisation gezweifelt werden könnte.

\section{Die Zerbrechlichkeit von Dyaden und die Auswirkung auf die Beratung}

Dyaden unterscheiden sich von anderen Rahmungen der Beratung dadurch, dass das Ausscheiden einer Person „das Ganze“ zerstören würde (vgl. Simmel, 1992, S. 101). Genauso wie der Tod eines Ehepartners, das Ausscheiden eines Geschäftspartners aus einer Beziehung oder die Aufkündigung eines ärztlichen Behandlungsauftrags durch den Patienten die Struktur der Dyade zerstört, endet auch eine dyadische Beratung, wenn entweder der Berater oder der Beratene sich zurückzieht. Hier unterschieden sich Dyaden von Gruppen, Familien, Organisationen oder Protestbewegungen, die sich zwar verändern mögen, wenn einzelne Personen ausscheiden, aber in der Regel dadurch in ihrer Existenz nicht unmittelbar in Frage gestellt werden (vgl. Becker \& Useem, 1942, S. 16).

Weil aus diesem Grund Dyaden besonders konfliktanfällig sind, kommt es häufig zu vorgreifenden Strategien der Konfliktmilderung. Man denke nur an das Liebesgesäusel von Paaren, die versuchen, Konflikte zu verhindern. Man findet aber auch ähnliche Prozesse in dyadischen Beratungssituationen. Die Rahmung einer Beratung ,unter vier Augen“ führt nicht selten dazu, dass sich häufig, gerade bei Konflikten des Klienten mit anderen Organisationsmitgliedern, eine Koalition zwischen Berater und Beratenem bildet. Der Berater wird dann als Koalitionspartner im Konflikt mit einer „bösen Umwelt" erlebt und als „,wichtige Unterstützung“ gegen Gegner innerhalb der Organisation wahrgenommen.

Aber trotz dieser Strategien der Konfliktmilderung kann es auch innerhalb der Dyaden zu Konflikten zwischen Beratern und Beratenen kommen. Diese Konflikte können sich ungewollt ergeben und können ein Zeichen für eine Krise in der Beziehung sein, sie können aber auch - ähnlich wie bei Therapien - die notwendige oder gar intendierte Folge der Beratung sein. Der Coach oder Supervisor legt bewusst den Finger in eine Wunde seines Klienten und riskiert damit einen Konflikt, oder er übernimmt die Position eines Gegners seines Klienten, um über den so initiierten Konflikt Material für die beraterische Arbeit zu sammeln.

Der Konflikt wird selbstverständlich durch die arbeitsbedingte Asymmetrie in der Beziehung zwischen Berater und Klient abgemildert. Genauso wie der Patient die Schmerzen, die ein Zahnarzt ihm zufügt, größtenteils mit der Rolle des Arztes erklären kann, besteht auch- 
für den Klienten eines Coachings oder einer Supervision die Möglichkeit, den entstehenden Konflikt auf eine professionelle Intervention des Beraters zurückzuführen. Und genauso wie der Zahnarzt die Beschimpfungen seines schmerzempfindlichen Patienten nicht auf sich persönlich zurechnen muss, braucht auch der Berater heftige Reaktionen des Klienten nicht als einen persönlichen Angriff zu verstehen. Aber trotz dieser Einschränkungen ist der Konflikt, der in einer dyadischen Beziehung ausbricht, ein Konflikt, der das Beratungsgespräch dominiert und so interessante Perspektiven für die Beratungsarbeit liefert.

Bei einer dyadischen Rahmung der Beratung kann dabei bewusst mit der Exit-Option beider Beteiligten gearbeitet werden. Der Klient wird durch den Konflikt in eine Situation gebracht, dass er sich entscheiden muss, ob er den Konflikt aushält oder die Arbeit abbricht. Und er weiß auch, dass auch der Berater immer die Möglichkeit hat, eine dyadische Beratungsbeziehung zu beenden, wenn er keine Fortschritte seiner Arbeit sieht. Weil beiden Seiten bewusst ist, dass der Austritt jedes Einzelnen die Dyade zerstört, entstehen Beratungsoptionen, die sich in anderen Rahmungen so sonst nicht ergeben würden.

\section{Gruppencoaching und Gruppensupervision: Speziell für Beratungen zusammengesetzte Gruppen}

Im Gruppencoaching und in der Gruppensupervision kommen Personen zusammen, die sich in vergleichbaren Arbeitssituationen befinden, im Alltag allerdings nicht zusammenarbeiten und die sich in der Regel vor dem Beginn der Beratung nicht kannten. Meistens besteht die Gemeinsamkeit der Gruppenmitglieder darin, dass ihre Arbeitssituation die gleiche ist. Alle Teilnehmer sind Führungskräfte oder Existenzgründer, Mobbingopfer verschiedener Unternehmen oder Doktoranden verschiedener Fakultäten einer Universität, Unternehmensnachfolger oder Freiberufler. Aber es gibt auch gerade in der Supervision den Ansatz, für Gruppenberatungen Personen aus unterschiedlichen Organisationen wie Schule, Pfarramt, Jugendamt, Krankenhaus oder Kindergarten zusammenzubringen (vgl. Belardi, 2002, S. 86).

Die Gruppensupervision entwickelte sich besonders ab den fünfziger Jahren des vorigen Jahrhunderts - inspiriert nicht zuletzt durch Erfahrungen mit den gruppendynamischen Experimenten Kurt Lewins in den USA und die Arbeiten des Mediziners Michael Balint mit Gruppen von Ärzten (vgl. dazu Mattke, 2006, S. 43f). Beide trugen dazu bei, dass bei der Beratung von Ärzten, Pfarrern, Lehrern und Sozialarbeitern die dyadische Rahmung der personenorientierten Beratung durch eine Beratung in Gruppen, die sich an Standards orientiert, ergänzt und teilweise auch ersetzt wurde.

Bei der Entwicklung der Gruppensupervision und - mit Abstrichen - auch des Gruppencoachings hat man auf vielfältige Erfahrungen mit Gruppenformaten in der Therapie zurückgegriffen: Die während des Zweiten Weltkrieges von Siegmund H. Foulkes im Laufe seiner Arbeit in einem Militärhospital entwickelte Methode einer psychoanalytischen Gruppentherapie spielt dabei ebenso eine Rolle wie die besonders von Wilfred R. Bion während und nach dem Zweiten Weltkrieg entwickelten Überlegungen zur Behandlung psychisch Kranker in Gruppentherapien.

Welche Mechanismen von Gruppen wirken jetzt in diesem Beratungsrahmen, und wie wirken sich diese aus? 
Was sind Gruppen, und welche Mechanismen wirken in ihnen?

Die Forschung über Gruppen hat lange Zeit darunter gelitten, dass der Begriff der Gruppe sehr unterschiedlich bestimmt wurde. Mal wurde dabei Gruppe als Synonym für jede Form von sozialem System verwendet, in dem Menschen „verknotet“ waren (vgl. Hofstätter, 1957, S. 177), mal als Kategorie, um eine Menge von Personen mit ähnlichen Merkmalen zu bezeichnen (z. B. die Gruppe der Chinesen in den USA oder die Gruppe der Zigarettenraucher). Mal wurde Gruppe nur im Sinne von Kleingruppe verstanden, also Personen, die in einem regelmäßigen, direkten, personenbezogenen Kontakt zueinander stehen, manchmal wurde der Begriff für jede Face-to-Face-Interaktion verwendet, in denen Menschen „miteinander in gegenseitigen Beziehungen stehen“ (vgl. Fichter, 1968, S. 69). Mal wurden auch komplette Organisationen unter dem Begriff der Gruppe gefasst, mal nur Abteilungen oder Cliquen in Organisationen (immer noch der beste Überblick und die beste Kritik des Begriffswirrwars findet sich bei König, 1983). Wenn im Folgenden von Gruppe die Rede ist, meine ich damit immer die Kleingruppe.

In der modernen Gesellschaft existiert eine Vielzahl unterschiedlicher gruppenartiger Zusammenschlüsse: in Form von Freundschaftskreisen (vgl. z. B. Schmidt, 2000), Cliquen pubertierender Jugendlicher (vgl. z. B. Uhlendorff \& Oswald, 2003), Straßengangs (vgl. Whyte, 1943), ,,autonomen“ linken politischen Gruppen (vgl. Binger, 1974), kleinen terroristischen Zusammenschlüssen wie der „Baader-Meinhof-Truppe“ (vgl. Rühmkorf, 2004, S. 38 ff.) oder religiösen Gruppierungen, die sich jenseits der Initiative von Kirchenorganen entwickelt haben (vgl. Lell \& Menne, 1976).

Bei der Betrachtung von Gruppen fällt auf, dass diese ein Gefühl von Zusammengehörigkeit entwickeln. Gruppen nehmen sich, so Niklas Luhmann in einer seinen frühen Ausarbeitungen, im alltäglichen Leben „bewusst als soziale Einheit wahr “ und grenzen sich so gegenüber ihrer Umwelt ab. Sie geben sich Namen, damit sie sich selbst mit einer Kurzformel identifizieren können und damit sie von anderen erkannt werden können, und sie nutzen Merkmale wie bestimmte Kleidung, Rituale oder Begrüßungssignale, um so ihre Grenzen zum „Rest der Welt" zu markieren (vgl. Luhmann, 1965, S. 176). ${ }^{\text {. }}$

Diese Ausbildung von Zusammengehörigkeitsgefühl setzt voraus, dass Gruppen - anders als beispielsweise Organisationen - aus einem bestimmten, unverwechselbaren Kreis von Personen bestehen. Zwar zerfällt eine Gruppe - anders als die oben beschriebenen Dyaden - nicht automatisch, wenn Personen aus der Gruppe ausscheiden oder neue Personen zu dieser Gruppe hinzustoßen. Aber sowohl die Kompensationsfähigkeit von Personenverlusten als auch die Aufnahmefähigkeit von neuen Personen sind in Gruppen begrenzt.

Das Gefühl von Zusammengehörigkeit entsteht in Gruppen - und auch da unterscheiden sie sich von Organisationen - auf der Basis eher diffuser Beziehungen zwischen ihren Mitgliedern. Während Organisationen wie Unternehmen, Verwaltungen oder Universitäten spezifische Rollenerwartungen an ihre Mitglieder stellen, bieten Gruppen Raum für vielfältigere Selbstdarstellungsmöglichkeiten. In Gruppen ist man nicht nur Schüler, nicht nur Sportler oder nicht nur Schläger, sondern letztlich ist fast alles, was eine Person betrifft, für Kommunikationen zugänglich (siehe dazu auch Neidhardt, 1979, S. 641 ff.; Neidhardt, 1983, S. 12 ff.; siehe auch Tyrell, 1983, S. 80; zur Unterscheidung „diffusspezifisch" siehe früh z. B. Parsons, 1939). 
Ähnlich wie in Familien, so ist auch in Gruppen das nicht spezifisch gruppenbezogene Verhalten ein legitimes Thema von Kommunikationen. Ein Gruppenmitglied kann die Anfrage nach einer neuen Liebesbeziehung nicht einfach mit der Aussage „Das geht Euch gar nichts an" abweisen. Es hat eine Auskunftspflicht auch für seine beruflichen Entscheidungen, selbst wenn die nicht unmittelbar für das Gruppengeschehen relevant sind. Während in Organisationen derjenige begründungspflichtig ist, der Auskünfte über das Verhalten über andere Rollen als die Organisationsrolle haben will, ist bei Gruppen - so könnte man einen Gedanken Talcott Parsons über Familien paraphrasieren - derjenige begründungspflichtig, der keine Auskunft über andere Rollen als die in der Gruppe geben will (vgl. dazu Luhmann, 1988, S. 182; Luhmann, 1990, S. 193).

Ebenso wie in Interaktionen, Familien, Protestbewegungen oder Organisationen bilden sich auch in Gruppen Normen aus. Das Besondere dieser Normbildungen ist jedoch - gerade im Vergleich zu Organisationen - dass sie nur in Ausnahmefällen explizit erfolgen. In Gruppen stehen in der Regel keine Verfahren zu Verfügung, um Normen zu ẩndern oder zu erweitern. Ihre Veränderungen finden deswegen eher unauffällig statt und sind manchmal Resultat von gruppendynamischen Zufällen (vgl. Tyrell, 1983, S. 80; siehe allgemein zu Gruppennormen Brown, 2000, S. 56 ff.).

Gruppen sind auf regelmäßige Interaktion angewiesen. In reiner Virtualität - bei einem völligen Verzicht auf Face-to-Face-Interaktionen - wird keine Gruppe überleben können. Aber Gruppen sind gleichzeitig mehr als eine Wiederholung von Interaktionen. Eine Gruppe verkraftet die Abwesenheit eines Gruppenmitglieds in einer Interaktion, ohne dadurch ihre Identität zu verlieren. Es wird bemerkt, wenn Lisa oder Hannes nicht bei einem Treffen dabei sind, aber die Gruppe wird dadurch nicht eine andere.

Gleichzeitig wird aber auch nicht jeder, der an einer Face-to-Face-Interaktion im Rahmen einer Gruppe teilnimmt, automatisch zum Gruppenmitglied. Ein Außenseiter kann mit einer Bande an einer Straßenecke abhängen und wird dadurch noch lange nicht zum Gruppenmitglied, genauso wenig wie der Forscher, der als teilnehmender Beobachter an einer Gruppensupervision teilnimmt, dadurch zum Mitglied der Gruppe wird (vgl. dazu Luhmann, 1969, S. 4 ff.).

\section{Zur Besonderheit von Gruppensupervisionen und Gruppenberatung}

Gruppen, die sich zum Coaching, zur Supervision oder auch zum gruppendynamischen Training treffen, unterscheiden sich offensichtlich von Freundschaftskreisen, Schulhofcliquen pubertierender Jugendlicher oder Straßengangs. Coachees oder Supervisanden kommen aufgrund eines allen Teilnehmern offensichtlichen Zieles zusammen. Es gibt keine Notwendigkeit, dass sich Teilnehmer dieser Gruppen jenseits dieses Zieles treffen, und wenn das Ziel erreicht ist, muss man neue Motive generieren, um sich noch zu treffen.

Aber in Gruppencoachings und Gruppensupervisionen (und besonders auch in gruppendynamischen Trainings) finden sich - und deswegen ist der Anschluss an die Soziologie der Gruppe hier relevant - viele der Elemente wieder, die man auch aus Freundschaftskreisen, Schulhofcliquen oder Straßengangs kennt: Ausbildung von Zusammengehörigkeit, Bemerken des Fehlens von Gruppenmitgliedern, Ausbildung eigener Gruppennormen oder Schwierigkeiten der Gruppenmitglieder, Fragen nach auch sehr persönliche Themen abzuweisen. 
In den Beratungssituationen werden jetzt diese Strukturierungsformen, die wir von Freundschaftskreisen, Schulhofcliquen oder Straßengangsgangs kennen, mobilisiert, um Lerneffekte bei den Gruppenmitgliedern zu erzielen (so ähnlich schon Nau, 1983, S. 126). ${ }^{2}$ Der Supervisor oder Coach als „Gruppenleiter“ nimmt dabei sicherlich eine Ausnahmestellung ein. Er unterliegt nicht den gleichen Verpflichtungen, sich den Gruppennormen zu unterwerfen, die Zugehörigkeitsrituale zu pflegen oder Persönliches von sich preiszugeben. Die Aufrechterhaltung dieser Differenz zu einfachen Gruppenmitgliedern ist im Gegenteil zentral, um die Gruppenprozesse noch steuern und bei Konfliktfällen eingreifen zu können. Jetzt wissen wir aber aus empirischen Studien über gruppendynamische Gruppen, dass diese Differenz zwischen Leitung und Gruppenmitglied immer wieder zusammenzubrechen droht. Die Position des Leiters war gerade in den auf Emanzipation ausgerichteten Gruppen prekär, und die Gruppe war häufig absorbiert durch die Dynamik des so genannten „Leitersturzes“ (vgl. Hirsch, 2004, S. 171 mit Verweis auf Slater, 1966).

Man kann die Effekte in Gruppencoachings und Gruppensupervisionen, aber auch weitergehend der Gruppentherapien oder gruppendynamischen Trainings, nur vor dem Hintergrund des Verschwindens von Gruppen als zentrales Ordnungsprinzip moderner Gesellschaften verstehen. In den vor 10000 Jahren verbreiteten Stammesgesellschaften in Europa, Afrika, Asien und Amerika war die Gruppe das zentrale Ordnungsprinzip. Bis zum Aufkommen der Hochkulturen waren Personen in Gruppen von 10 bis 100 Personen organisiert, in denen sich alle Gruppenmitglieder kannten und sowohl den Eintritt als auch das Ausscheiden von Personen von allen bemerkt wurde (vgl. Tenbruck, 1972, S. $56 \mathrm{ff}$.).

In der modernen Gesellschaft haben Gruppen jedoch ihre zentrale Bedeutung bei der Strukturierung der Gesellschaft verloren. Die moderne Gesellschaft wird viel stärker durch Organisationen geprägt. Wer weder in der Schule gewesen ist noch beim Miliär gedient hat, keinen Job gefunden hat und keinem Verein angehört hat, von dem könnte, so pointiert Jürgen Kaube (2000, S. 62), wohl mit Recht gesagt werden, er lebe am Rande der Gesellschaft.

Die gleiche Bedeutung lässt sich Gruppen in der modernen Gesellschaft nicht mehr zuweisen. Man kann seine Adoleszenzphase durchleben, ohne Mitglied einer Freundesclique zu sein, und man kann regelmäßig ins Fußballstadion gehen, ohne sich einer sich selbstorganisierenden Gruppe von Hooligans anzuschließen. Eine Karriere in der Politik ist möglich, ohne dass man einer festen Clique von Parteigenossen angeschlossen ist. Als Vorstandsvorsitzender eines DAX-Unternehmens mag es förderlich sein, mit einer Clique anderer Vorstandsvorsitzender unter Führung von Reinhold Messner regelmäßig Berge zu erklimmen, aber eine Karriere hängt nicht von der Zugehörigkeit zu dieser Clique ab und wird vielleicht noch nicht einmal dadurch gefördert.

Erst vor dem Hintergrund dieses Bedeutungsverlustes der Gruppe für die moderne Gesellschaft lässt sich jetzt erklären, weswegen in Gruppensupervisionen oder Gruppencoachings, besonders aber auch in Gruppentherapien und gruppendynamischen Trainings, überraschende Lerneffekte erzeugt werden können. Personen können sich in der modernen Gesellschaft Gruppenprozessen so leicht entziehen, dass die Dynamik in Gruppen, so die These, mit ihrer Diffusität der Beziehungen und mit der Ausbildung von Zusammengehörigkeitsgefühlen eine überraschende Erfahrung darstellt. 
Was ändert sich, wenn eine personenorientierte Beratung in Gruppen stattfindet?

Mit der Beratungsarbeit in Gruppen wurde spätestens seit den siebziger Jahren des vorigen Jahrhunderts eine Vielzahl von Hoffnungen verknüpft. Die Arbeit mit Gruppen wurde als Kern einer emanzipatorischen Veränderung der Gesellschaft begriffen. Die Arbeit mit T-Gruppen, so eine der vielfältigen Hoffnungen, könnte zur Lösung nationaler, aber auch internationaler Konflikte führen (vgl. z. B. Doob, 1970). Die Arbeiter, Angestellten und auch Manager in Verwaltungen, Unternehmen, Krankenhäusern, Universitäten und Kirchen sollten durch gruppendynamische Trainings aus den Entfremdungszwängen befreit werden. Die Gruppe galt, so schon der Untertitel eines populären Buches von HorstEberhard Richter (1972) - als ein ,neuer Weg, sich selbst und andere zu befreien“. Die Hoffnung war, dass „die Gruppe“ den „etablierten Autoritäten“ in Politik, Wirtschaft, Wissenschaft, Recht und Medizin die Macht entreißen könnte und durch eine „gerechtere, legitimere Form der Machtausübung“ ersetzen könnte (vgl. Edding, 2005, S. 5; siehe auch Hirsch, 2004, S. 170; siehe besonders die frühe, immer noch lesenswerte Kritik von Horn, 1969).

In den Selbstbeschreibungen von Coaches und Supervisoren finden sich - sicherlich korrekterweise - Hinweise auf die Möglichkeit, in Gruppen eine Vielzahl von Perspektiven zu mobilisieren. Durch diese Vielzahl verschiedener Perspektiven könnten Probleme in der Gruppe häufig besser gelöst werden, als wenn dies eine einzelne Person macht (vgl. Wetzel, 1998, S. 113 ff.). Die Gruppe erziele deswegen nicht nur als Gruppe gute Ergebnisse, sondern wirke auch als ,Vermittlerin individueller Lernprozesse“" (vgl. Huber \& Müller, 1998, S. 218 ff.).

Soziologisch ist für den Erfolg (beziehungsweise auch für den Misserfolg) der gruppenzentrierten Beratung jedoch eher der Gruppenzwang relevant. Dabei sind die Zwänge der Gruppe nicht als Gegensatz zur Freiwilligkeit der Teilnahme an der Gruppe zu verstehen, sondern die Zwänge der Gruppe bilden sich erst auf der Basis der Freiwilligkeit aus. Das Besondere der Beratung in Gruppen ist, dass die Person durch eine eigene Entscheidung teilnimmt. Aber genau diese eigene Entscheidung erschwert es einer Person, die an einer Beratung in der Gruppe teilnimmt, sich zu entziehen. Weil die Teilnahme an der Sitzung nicht erzwungen wurde, sondern freiwillig erfolgt, binden sich die Mitglieder an eigene Entscheidungen. Sie ,verlieren ihr Gesicht“, wenn sie kurz nach der Erklärung zur Bereitschaft, an einer Gruppensitzung teilzunehmen, aufgrund der ,Zwänge der Gruppe“ wieder aussteigen. ${ }^{3}$

Wodurch wird in der Gruppe jetzt der Zwang aufgebaut?

\section{Über die Funktion des Dritten}

Mit Georg Simmel lässt sich eine Vielzahl von Effekten in Gruppen auf die Existenz eines Dritten zurückführen. Mit dem Dritten wird die Person bezeichnet, die aus der Dyade eine regelmäßig zusammenkommende Gruppe macht (vgl. Wiese, 1966, S. 466 ff.), die eine Liebesbeziehung in eine Familie mit eigenen Regeln und Gesetzmäßigkeiten umwandelt, die mit der Zweierbeziehung wenig zu tun hat (vgl. Allert, 1997, S. 31 ff.), oder die einem Gespräch zweier Personen allein durch ihre Anwesenheit eine ganz eigene Dynamik verleiht (vgl. Goffman, 1971, S. 83 ff.). Natürlich kann immer noch ein Vierter, Fünfter 
oder Sechster hinzukommen, aber dadurch, so das Argument Simmels, verändert sich das soziale Gebilde nicht mehr prinzipiell (vgl. Simmel, 1992, S. 124).

In der Simmelschen Tradition wurde eine Vielzahl unterschiedlicher Funktionen identifiziert, die ein Dritter ausüben kann. Der Dritte kann sich beispielsweise als unparteiischer Vermittler profilieren - entweder dadurch, dass er von den anderen dafür formal benannt wird oder dadurch, dass er sich die Rolle durch konkretes Verhalten in der Interaktion aneignet (vgl. Simmel, 1992, S. 125 ff.). Der Dritte kann aber auch frei nach dem Motto „Wenn zwei sich streiten, freut sich der Dritte“ versuchen, einen Vorteil daraus zu ziehen, dass sich die anderen beiden wechselseitig aufreiben (vgl. Simmel, 1992, S. $134 \mathrm{ff}$.). Das kann bis hin zu Strategien des ,Teile und Herrsche“ führen, indem der Dritte „Zwist vorsätzlich stiftet, um eine beherrschende Situation zu gewinnen“ (vgl. Simmel, 1992, 143). Der Dritte kann auch als Intrigant versuchen, mittels geheim gehaltener böser Nachrede eine mächtige Person in einer Dreierbeziehung dazu zu bringen, gegen ein Intrigenopfer vorzugehen (vgl. ausführlich Utz, 1997, S. 19 ff.).

Auffällig bei dieser Sammlung von Funktionen des Dritten in der Tradition Simmels ist, dass vorrangig die „Streitformen“ aufgefuihrt werden, die durch den Dritten entstehen (vgl. Freund, 1976, S. 90 ff.). Die Betonung der „Streitformen“ macht sicherlich Sinn, wenn man wie Simmel vorrangig das Verhältnis von Personen, Organisationen oder Staaten zueinander im Blick hat (vgl. Simmel, 1992, S. 284 ff.). Und auch innerhalb von Gruppen lassen sich solche „Streitformen" sicherlich beobachten (und werden ja gern in gruppendynamischen Trainings aufgegriffen), aber für die Analyse der Funktion des Dritten in Gruppen sind meines Erachtens die Eintrachts- und Gemeinschaftsfunktionen des Dritten wichtiger.

Für die Untersuchung von Gruppensupervision und Gruppencoaching ist es zentral, dass sich erst durch den Dritten Mehrheiten bilden können. In einer „Vereinigung von Zweien“ gibt es, so Simmel, „keine Majorität“. Aber schon durch den „Hinzutritt eines Dritten“ ist die Gelegenheit dazu gegeben (vgl. Simmel, 1992, S. 116). Anstelle eines „Verhältnisses $1: 1$ “ bei der Dyade kann es durch den Dritten nun zu einem ,Verhältnis 2 : 1 “ kommen. Während bei der Dyade Normen immer mit dem Verweis zurückgewiesen werden können, dass diese doch lediglich die Position des anderen darstellen, erhalten sie allein durch das „Verhältnis $2: 1^{\text {“ }}$ eine ganz eigene Form von Legitimität (vgl. Aubert, 1972, S. 194 f.). Besonders in Gruppen, die ja keine formale Hierarchie kennen, bilden sich so Mehrheiten, die der Minderheit über die Legitimität des Dritten ihre Interpretationen mit einem gewissen Nachdruck vermitteln können.

Durch die Mehrheitsbildung in Gruppen kann die Asymmetrie, die sowieso schon zwischen Berater einerseits und Klienten andererseits existiert, noch weiter verstärkt werden. Die in Gruppen stattfindende personenorientierte Beratung profitiert davon, dass der jeweils im Mittelpunkt stehende Klient einem Ensemble bestehend aus dem Coach oder Supervisor sowie allen anderen Gruppenmitgliedern gegenübersteht. Diese Ensembles bilden sich automatisch aus, wenn in einer Gruppe das Problem eines der Klienten besprochen wird, sie können aber auch in der standardisierten Form eines ,heißen Stuhls“ gezielt eingerichtet werden, indem alle anderen Beteiligten einer in der Mitte sitzenden Person ihren Eindruck schildern. Das Ensemble bildet in der Interaktion eine „Kollusion“, die jedenfalls für die kurze Phase, in der der Klient Thema ist, die Situationsdefinition dieses Klienten bestimmt (vgl. Goffman, 1982, S. 438). 
Es ist meines Erachtens dieser durch den Dritten (und Vierten und Fünften) erzeugte Druck, der dazu führt, dass durch Gruppen überraschende Effekte auf Einzelpersonen erzielt werden können. Dabei spielen vermutlich nicht nur vorrangig die Möglichkeiten zur Selbstverwirklichung eine Rolle, sondern besonders der von der Gruppe aufgebaute Zwang. Diese Zwänge der Gruppe sind maßgeblich dafür verantwortlich, dass überhaupt so etwas wie ein „Persönlichkeitsumbau“ von erwachsenen Menschen stattfinden kann.

\section{Alltäglich kooperierende Teams: Team-Coaching und Team-Supervision}

Supervision und Coaching von Teams unterscheiden sich von den oben angesprochenen Gruppencoachings und Gruppensupervisionen dadurch, dass die Personen sich nicht nur in der Beratungssituation treffen, sondern auch im Alltag zusammenarbeiten. Bei der Teamsupervision oder beim Teamcoaching kann es sich entweder um Beratung für monoprofessionelle Teams handeln, die sich aus Teammitgliedern mit weitgehend gleicher Ausbildung zusammensetzen, oder um Beratung von multiprofessionellen Teams, in denen beispielweise in einem Krankenhaus Ärzte, Psychologen, Sozialarbeiter, Heilpädagogen und Krankenschwestern zusammenwirken (vgl. dazu Belardi, 2002, S. 94).

Auf den ersten Blick haben Teams viele Gemeinsamkeiten mit den jenseits von Organisationen existierenden Gruppen wie Freundschaftscliquen, Straßengangs oder „autonomen“ linken politischen Gruppen. Auch bei ihnen kennen sich die Personen untereinander. Es wird bemerkt, wenn Personen dazukommen oder ausscheiden. Aber Teams existieren - und das ist der zentrale Unterschied - nur im Rahmen von Organisationen und können deswegen nicht die gleiche Form von Autonomie ausbilden, die Gruppen außerhalb von Organisationen entwickeln. Um diese Abstufung der Autonomie zu markieren, werden Teams und Cliquen, die sich innerhalb von Organisation ausbilden, häufig auch als Gruppierung und nicht als Gruppe bezeichnet.

Bezüglich der Begrifflichkeit gibt es innerhalb der Wissenschaft keine einheitliche Bestimmung. In Kenntnis konkurrierender Definitionsversuche wird hier folgende Begrifflichkeit vorgeschlagen. Bei „Gruppen“ handelt es sich um eigenständige soziale Systeme, die über ihre Mitglieder selbst entscheiden können, sich ihre Regeln weitgehend selbst geben und sich dadurch von ihrer Umwelt abgrenzen. Innerhalb von Organisationen existieren „Gruppierungen“, die dadurch gekennzeichnet sind, dass sie zwar Merkmale von Gruppen aufweisen, aber aufgrund ihrer Einbindung in die Organisation nur begrenzte Autonomie haben. Dabei können zwei Typen von Gruppierungen unterschieden werden: Einerseits „Teams“, die durch formale Entscheidungen der Organisation gebildet werden, und andererseits „Cliquen“, die sich in der Informalität der Organisation bilden.

Wie rigide diese Definition ist, zeigt, dass von den sechs „Beispielgruppen“, die George Caspar Homans (1950) vorschlägt, nur die Norton Street Gang eine Gruppe im engeren soziologischen Sinne wäre. Beim Bank Wiring Observation Room, bei der Spitze der Elektrogerätefirma und den „Verwaltungsleuten“ würde es sich um Teams handeln, die nur durch eine Soziologie der Organisation erklärt werden könnten. Die polynesische Insel Tikopia mit 1200 Einwohnern und der Kleinstadt Hilltown würde man als Ganzes weder mit den Begriffen der Gruppe noch mit denen von Teams oder Cliquen fassen können. 
Teams und Cliquen - Was zeichnet Gruppierungen in Organisationen aus?

In Organisationen müssen also zwei unterschiedliche Formen von Gruppierungen grundsätzlich auseinander gehalten werden. Die erste Form der Gruppierung - die in Anlehnung an den üblichen Sprachgebrauch als Team (oder früher als Arbeitsgruppe) bezeichnet werden kann, entsteht durch eine Entscheidung der Organisation. Es handelt sich um kleine Abteilungen, Service-Teams, Steuerungskreise in Unternehmen, Züge in Panzer-Kompanien, teilautonome Fertigungs- oder Montagegruppen oder auch Vorstände, die sich auf die Formalstruktur der Organisation berufen können und in ihrer weiteren Existenz auch von formalen Entscheidungen der Organisation abhängen. Sie sind in den Organigrammen der Organisation vermerkt, sie finden sich in den Akten wieder, und man kann auf sie in den Protokollen der Organisation Bezug nehmen.

Die Eingebundenheit dieser Teams in die Organisation hat schwerwiegende Folgen. Die Teams in Organisationen können über ihre Mitgliedschaft nicht selbst verfügen. Eine kleine Arbeitsgruppe hat keine formalen Möglichkeiten, sich dagegen zu wehren, wenn ihr eine neue Mitarbeiterin zugewiesen wird. Ein über längere Zeit eingerichtetes Projektteam kann nicht autonom darüber entscheiden, welche Personen Mitglied dieses Teams sein sollen und welche nicht. Dadurch sind sie nicht in der Lage, eigene formale (!) Normen zu entwickeln und durchzusetzen. Eine teilautonome Montagegruppe in der Automobilindustrie kann keine Regeln aufstellen, die beispielsweise dem Regelwerk der Gesamtorganisation widersprechen, und ihre Teammitglieder dann mit Verweis auf die riskante Mitgliedschaft im Team auf diese Regeln einschwören (siehe früh schon Luhmann, 1965, S. 176).

Dies führt - im Vergleich zu den oben dargestellten Gruppen - dazu, dass Teammitglieder in Organisationen immer nur in ihrer Rolle als Organisationsmitglied auftreten müssen. Man kann über Jahre Kollegen in einer Abteilung haben, von denen man nicht weiß, ob sie Affären haben, ob ihre Eltern noch leben und welchen Basketballverein sie ihrer Freizeit unterstützen. Und weitergehend: diese Kollegen können mögliche Anfragen ihrer Teammitglieder bezüglich dieser Themen zurückweisen und sich dabei im Recht fühlen.

Es ist selbstverständlich nicht ausgeschlossen, dass sich in Teams auch persönliche Beziehungen ausbilden können (ganz selten aber persönliche Beziehungen aller Mitglieder eines Teams zueinander). Aber die Organisation ist - wie es teilweise im Ansatz der Human Relations suggeriert wird - auf diese persönlichen Beziehungen nicht angewiesen, damit die Mitglieder ihre Leistungen erbringen. Straffe Leitung, hohe Entlohnung und symbolische Abstrafung einzelner Mitglieder können ähnliche Effekte erzielen (vgl. Luhmann, 1964, S. 107).

Mit Cliquen existiert in Organisationen eine zweite Form der Gruppierung, die sich jenseits der Formalstruktur einer Organisation bildet. Cliquen entstehen in ,natürlicher Fortsetzung und Verdichtung kollegialer Beziehungen“, und zwar immer dort, wo das Verhältnis zur formalen Organisation besonders distanziert und problematisch ist. Bei Cliquen handelt es sich also, kurz ausgedrückt, um Zusammenschlüsse von Mitarbeitern, die jenseits ihrer formalen Aufhängung in der Organisation zusammenwirken (vgl. Luhmanns, 1964 S. 324). 
Niklas Luhmann unterscheidet verschiedene Ausrichtungen von Cliquen, die teilweise kombiniert werden. Die erste Ausrichtung - in Reinform bei der Clique der Unzufriedenen - dient zur „Erhaltung der Selbstachtung“, besonders in Situationen, die von ihren Mitgliedern als bedrohlich empfunden werden. Wenn Organisationsmitglieder den Eindruck haben, dem Druck innerhalb einer Organisation nicht mehr gewachsen zu sein, dann reicht der ,kollegiale Halt“ häufig nicht mehr aus, und es bilden sich Gruppierungen von missmutigen oder resignierenden Mitgliedern aus. Die zweite Ausrichtung - besonders auffällig zu finden bei so genannten Konversationscliquen - dient vorrangig dazu, jenseits des formal gebotenen und immer wieder eingeforderten Konsenses in einer Organisation sich über die „wirkliche Situation“ zu verständigen. Bei der dritten Ausrichtung geht es den Cliquenmitgliedern vorrangig darum, sich in ihren persönlichen Karriereinteressen wechselseitig zu unterstützen. Es handelt sich in der Reinform um Seilschaften wie dem Andenpakt von CDU-Politikern, die dazu dienen, jenseits der formalen Strukturen das Fortkommen der Cliquen-Mitglieder zu fördern. Bei der vierten Ausrichtung geht es darum, jenseits der formalen Struktur der Organisation Ziele durchzusetzen, die durch die Organisation noch nicht positiv sanktioniert werden. „Strategischen Cliquen“ geht es dabei nicht vorrangig um die individuelle Karriere ihrer Mitglieder, sondern um das Erreichen von Zielen, die offiziell noch nicht auf der Agenda der Organisation vertreten sind (vgl. ausführlich Luhmann, 1964, S. 325).

Cliquen unterscheiden sich von Teams dadurch, dass sie keine genau definierten Grenzen der Mitgliedschaft haben. Es ist häufig gar nicht klar, wer zu einer Clique in Organisationen dazu gehört und wer nicht. Die Übergange zu ganz normalen kollegialen Beziehungen, darauf weist Luhmann hin, sind fließend. Die Existenz von Cliquen in Organisationen ist immer prekär. Sie sind anfällig gegen den Wechsel von Personen und zerfallen häufig, wenn auch nur eine oder zwei Personen die Organisation verlassen. Da es - anders als bei Teams - keine durch die Organisation abgesicherte Zuweisung von Personen zu Cliquen gibt, ist die Identität der Clique - für die Cliquenmitglieder und erst recht für Außenstehende - häufig nur schwer zu fassen (zu all diesen Punkten ausführlich Luhmann, 1964, S. 331 f.; Luhmann, 1965, S. 175 f.).

Weil nur die erste Form von Gruppierungen in Organisationen durch die Formalstruktur abgesichert ist, finden wir Coaching und Supervision fast ausschließlich bei diesem Typus von Gruppierungen in Organisationen. Coaching oder Supervision für Cliquen, Seilschaften oder informelle Gruppierungen sind seltene Fälle. Da diese ja nur quasi im Schatten der Formalstruktur der Organisation existieren, sind sie nur begrenzt in der Lage, eine formal wirkende Entscheidung wie die Beauftragung eines Beraters zu treffen oder auch nur die notwendigen Mittel für eine solche Beratung zu mobilisieren. ${ }^{4}$

Für beide Gruppierungen in Organisationen - Teams und Cliquen - gilt aber, dass sie nicht die gleiche Form von Autonomie entwickeln wie die Gruppen, die sich außerhalb von Organisationen bilden. Lediglich für den Fall der Gruppen, die sich aufgrund von diffusen Beziehungen zwischen Personen außerhalb von Organisationen bilden, dürfte man aus systemtheoretischer Perspektive den Systembegriff herausrücken. Oder in der sprachlich anspruchsvollen, aber nicht unbedingt notwendigen Formulierung: Nur bei Gruppen handelt es sich um autopoietische, selbstreferentielle Systeme, die sich in Differenz zur Umwelt konstituieren. Teams und Cliquen können lediglich als Subsystem eines autopoietischen, selbstreferentiellen Systems - nämlich der Organisation - verstanden werden. 
Man könnte argumentieren, dass die bisher nicht besonders fruchtbare systemtheoretische Diskussion, ob Gruppe ein soziales System ist oder nicht, an dieser Stelle nicht unbedingt zu interessieren braucht. Es gehört zu einer der Pathologien systemtheoretischer Debatten, dass sich man sich in Definitionsschlachten darüber erschöpft, welche sozialen Phänomene ein soziales System sind und welche nicht. Aber gerade in der Unterscheidung von Gruppensupervision und Gruppencoaching einerseits und Teamsupervision und Teamcoaching andererseits kann man unmittelbar Gewinn aus einer präzisen Bestimmung von Gruppen ziehen. Und so macht es einen großen Unterschied, ob es sich bei den Klienten eines Coachings oder einer Supervision um eine Gruppe handelt, die sich lediglich zu dem Zwecke dieser Beratung trifft, oder ob es sich um Teams handelt, die im Rahmen einer Organisation gebildet wurden.

Gruppen, die sich nur zum Zwecke der Beratung treffen, haben teilweise die Möglichkeit, über ihre Mitglieder selbst zu entscheiden, können sich ihre Regeln selbst geben und sich auf eigene Initiative hin auslösen. Das eröffnet gerade für die Beratung in Gruppen eigene Möglichkeiten. Dagegen haben Teams nur sehr begrenzte Möglichkeiten, darüber zu entscheiden, wer zu ihnen gehört, welche Regeln gelten und wie lange sie existieren. Teams erhalten dagegen durch die Organisationen eindeutige Mitgliedschaftskriterien vorgegeben, und ihr Fortbestand ist trotz Mitgliederwechsel garantiert. ${ }^{5}$ Das wirkt sich automatisch auf die Möglichkeiten der Beratung in Teams aus.

Welche besonderen Effekte treten bei Teams in Organisationen auf?

Aus soziologischer Perspektive ist interessant, dass bei der Behandlung von personenbezogenen Themen in Teams - anders als bei Gruppen - die organisationsinterne Öffentlichkeit als ein Zensurmechanismus auftreten kann. Mit Zensurmechanismus ist gemeint, dass einzelne Teammitglieder (oder auch das ganze Team) Informationen zurückhalten, die durch das Teamcoaching oder die Teamsupervision eigentlich herausgelockt werden sollen. Man erzählt im Coaching nichts über Probleme zwischen zwei Teammitgliedern, obwohl dies vom Coach erwartet wird oder hält - trotz Nachfrage - in der Supervision Informationen über Empfindungen in einer problematischen Situation zurück.

Für diesen Zensurmechanismus in der Teamsupervision und im Teamcoaching gibt es zwei Gründe. Der erste Grund ist das Gesetz des Wiedersehens, das in jeder Organisation herrscht. Den gleichen Personen, denen man in der Teamsupervision oder im Teamcoaching begegnet, wird man mit Sicherheit wieder begegnen. Das Besondere ist aber, dass die Situationen, in denen man den Personen wieder begegnen wird, in der Regel andere sind als die eher außergewöhnliche Beratungssituation im Teamcoaching oder in der Teamsupervision. So droht man beim zufälligen Zusammentreffen mit einem Teamkollegen am Kopierer daran erinnert zu werden, dass man in der letzten gemeinsamen Sitzung Teile seiner Persönlichkeit gezeigt hat, an die man am Kopierer lieber nicht erinnert werden möchte. In Antizipation des Wiedersehens halten sich Teammitglieder dann zurück.

Der zweite Grund ist die Existenz ,gemeinsam bekannter Dritter“ außerhalb des Teams. Während man in den häufig schon fast mystisch verklärten, zufälligen Zusammenkünften in Eisenbahnabteilen meint alles erzählen zu können, weil man ja mit den Zufallsbekanntschaften keine gemeinsamen Bekannten hat, ist jedem Teilnehmer einer Teamsupervision 
und eines 'Teamcoachings bewusst, dass es außerhalb der Sitzung Personen gibt, die allen Teilnehmern bekannt sind. Dies führt dazu, so André Kieserling (2002), dass in den Sitzungen immer der Verdacht mitschwingt, dass „man auf Vertrauen und Diskretion nicht zählen" kann. Bei informellen Treffen mit Kollegen und manchmal auch mit Vorgesetzten könne man sich auf diese ,wertvollen Ressourcen“ verlassen, in offiziellen Sitzungen der Teams würden diese Ressourcen aber allen zugänglich gemacht werden.

Bei der Betrachtung von Teamsupervisionen oder Teamcoachings - weitergehend aber auch von Team-Workshops generell - ist die Frage, inwiefern die Eigendynamik von Face-to-Face-Interaktionen diese Zensurmechanismen außer Kraft setzt. Es können sich - so die Vermutung - gerade bei Teamsupervisionen und Teamcoachings Ermüdungserscheinungen gegenüber der Phrasendrescherei ausbilden, die zu einem Zwang zur Offenheit führen, den man vielleicht später bereut.

\section{Fazit: Die Spannungsfelder der personenorientierten Beratung in den unterschiedlichen Rahmungen}

In den drei verschiedenen Beratungsrahmen lassen sich vier verschiedene Ziele unterscheiden, die durch die Supervision oder das Coaching angestrebt werden. Als erstes Ziel von Beratung kann die personenorientierte Beratung einer Person angesehen werden. Dieser Aspekt spielt besonders in dyadischen Beratungsbeziehungen eine wichtige Rolle, weil der Beratene dort die volle Aufmerksamkeit des Coaches oder Supervisors erhalten kann und er davon ausgehen kann, dass seine Person im Mittelpunkt jeder einzelnen Beratungssitzung steht. Aber die Beratung einer einzelnen Person kann auch in einer Gruppe stattfinden. Die Gruppe oder das Team tritt dabei quasi als eine Art „Gesamtsupervisor" oder "Gesamtcoach" für die einzelne Person auf (vgl. Oevermann, 2001, S. 270). Auch für die Teamsupervision und das Teamcoaching wird der Anspruch aufgestellt, dass hier eine Beratung einer einzelnen Person stattfinden kann.

Das zweite Ziel betrifft die Verbesserung der Leistungen für einen Klienten der Organisation. Die Ursprünge der personenorientierten Beratung liegen, wie gezeigt, in der Fallberatung, die voranging und auf einen abwesenden Dritten - den Klienten des Supervisanden oder Coachees - ausgerichtet ist. Die Fallberatung kann dabei in einer dyadischen Rahmung erfolgen - ein Arzt schildert das Problem mit einem Patienten oder ein Coach in der Ausbildung sein Problem mit einem Coachee. Die Fallberatung kann aber auch, wie das Beispiel der Balintgruppen zeigt, in Anwesenheit einer Reihe anderer Professionellen erfolgen, die es in ihrer Praxis mit ähnlichen Fällen zu tun haben. Bei einer Teamsupervision wird dann unter Anwesenheit eines Beraters ein in der Regel allen Teammitgliedern bekannter Fall eines Klienten behandelt und gemeinsam überlegt, wie in diesem Fall vorgegangen werden sollte.

Ein drittes Ziel, das sich im Rahmen von Coachings und Supervisionen ausbilden kann, besteht in einer Verbesserung der Zusammenarbeit von Personen im Team. Diese Beratung kann prinzipiell auch in der dyadischen Rahmung erfolgen, zum Beispiel wenn der Klient mit seinem Berater darüber reflektiert, wie er auf seine unmittelbaren Arbeitskollegen wirkt. In der Regel wird dieses Ziel bei Gruppencoachings- und Gruppensupervisionen oder bei Teamcoachings oder Teamsupervisionen angestrebt. Bei der Beratung 
in Gruppen wird in der Tradition der Gruppendynamik davon ausgegangen, dass die für den Beratungskontext künstlich gebildeten Gruppen Strukturähnlichkeiten zu den Teams haben, in denen die Klienten regelmäßig arbeiten und insofern ein Lerntransfer in den realen Arbeitsalltag stattfinden kann. Bei der Teamsupervision und dem Teamcoaching bestehen fließende Übergänge zum Teamtraining. Hier wird die Zusammenarbeit im Team selbst thematisiert in der Hoffnung, dass die Selbstreflexion zu einer Verbesserung der Arbeitsleistungen führt.

Der vierte Aspekt, der gerade in der aktuellen Praktikerliteratur hervorgehoben wird, ist die Möglichkeit, Coaching und Supervision als Teil einer Organisationsentwicklungsstrategie zu betreiben. Dies kann in einer dyadischen Rahmung stattfinden, beispielsweise wenn der Geschäftsführer eines Familienunternehmens eine Supervisorin als Sparringspartnerin dafür nutzt, um eine angedachte neue Strategie zu prüfen. Eine ähnliche Funktion könnte man auch bei der Gruppensupervision und dem Gruppencoaching erkennen, wenn ein Gruppenmitglied Überlegungen zur Veränderung seiner Organisation zur Diskussion stellt. Am stärksten wird dieses Ziel jedoch beim Teamcoaching und bei der Teamsupervision betont. Die Beratung des Teams ist dabei dann vorrangig Mittel zum Zweck, um die Organisation insgesamt leistungsfähiger zu gestalten (vgl. früh schon Rappe-Giesecke, 1990; Schreyögg, 1991; Buchinger, 1992; siehe auch Fatzer, 1999).

Häufig wird in der Debatte implizit immer noch davon ausgegangen, dass sich die vier Ziele gut kombinieren lassen. Die Fallsupervision könnte, so der Vorschlag, gut mit einer personenorientierten Beratung einer einzelnen Person verbunden werden. Das Coaching oder die Supervision eines Teams könnte, so eine andere Idee, verknüpft werden mit der personenorientierten Beratung einer einzelnen Person. Und diese verschiedenen Ansätze - die Beratung konkreter Fälle, die Beratung einzelner Personen und die Beratung von Teams - könnte dann wiederum im Sinne einer ,vernetzten Beratung“ mit Maßnahmen der Organisationsentwicklung gekoppelt werden.

Hinter diesen Konzepten zur Vernetzung unterschiedlicher Zielsetzungen personenorientierter Beratung versteckt sich meines Erachtens ein zu harmonistisches Organisationsverständnis. Es wird letztlich suggeriert, dass das, was gut ist für eine Beratung von Einzelpersonen, auch gut für die Beratung des Teams ist und das, was gut für das Team ist, auch gut für die Organisation ist. Spätestens seit der Kritik an dem Human Relations Ansatz der sechziger Jahre des letzten Jahrhunderts gibt es für ein solch ein Organisationsverständnis aus organisationswissenschaftlicher Perspektive kaum noch Unterstützung. Ins Blickfeld einer organisationswissenschaftlichen Betrachtung geraten eher die Konkurrenzen, die sich zwischen den vier Zielen der personenorientierten Beratung ausbilden.

Diese Debatte wird im Coaching und in der Supervision unter dem Begriff der ,,verschiedenen Auftraggeber“ geführt. Auch wenn der Coach oder Supervisor von der Organisation beauftragt wird, eine personenorientierte Beratung durchzuführen, dann ist damit noch nicht festgelegt, wem gegenüber er sich im Konfliktfall verpflichtet fühlt: dem Klienten, an dem er die Beratungsleistung erbringt? Dem Team, in das der Klient eingebunden ist? Der Personalentwicklungsabteilung, über die er eventuell vermittelt wurde? Einem abstrakten Etwas, wie der Organisation an sich? Oder vielleicht auch nur seinem eigenen Beratungsverständnis (vgl. beispielsweise Feltham \& Dryden, 2002, S. 20 ff.)? 
Der Blick auf die drei unterschiedlichen Rahmungen der Beratungen eröffnet die Perspektive dafür, dass sich in ihnen die vier Ziele unterschiedlich gut umsetzen lassen. Die Beratung einzelner Personen droht im Rahmen von Teams oder Gruppen zu einer „Einzelberatung unter Zeugen“ zu degenerieren (vgl. Looss, 1997, S. 155). Gleichzeitig ist es aber fraglich, ob bei der dyadischen Beratung - der „Einzelberatung ohne Zeugen“ - Effekte erzielt werden können, die Rückwirkungen auf die Arbeit in einzelnen Teams oder gar der ganzen Organisation haben. Der Selbstzensurmechanismus, der sich in Teams einstellt, könnte wiederum erklären, weswegen die Diskussion über Probleme mit den Klienten der Supervisanden und Coachees besonders anbieten, gewährleistet die Referenz auf einen abwesenden Dritten doch immer einen gewissen Schutz für Personen. Die systematische Debatte über diese Frage beginnt hier erst, ohne dass sich bisher eine eindeutige Position unter Praktikern abzuzeichnen scheint.

\section{Anmerkungen}

1 Die hier zitierte Stelle von Luhmann ist interessant, weil sie so gelesen werden kann, dass er - jedenfalls in dieser frühen Phase - sehr wohl anerkennt, dass Gruppen soziale Systeme mit eigener Logik sein können. In dem kurzen Abschnitt spricht er sich zwar gegen die Inflation des Gruppenbegriffs in den fünfziger und sechziger Jahren aus. Vieles, was als Gruppe bezeichnet wird, seien, so Luhmann, lediglich „Kontaktsysteme“" oder einfache „Interaktionen“. Aber statt ganz auf den Gruppenbegriff zu verzichten, plädiert er dafür - so jedenfalls meine Lesart -, den „Gruppenbegriff für eine spezifische Situation“ zu reservieren. Zentral scheint dabei das für Gruppen typische Element zu sein, dass einige Personen zu den Mitgliedern der Gruppe gezählt werden können und andere nicht und dass die Gruppe auch erhalten bleibt, wenn einzelne Personen wechseln. Als systemtheoretischer State of the Art zur Gruppe wird zurzeit immer noch die prinzipielle Ablehnung der Gruppe als soziales System gehandelt. Als Referenzen gelten dabei neben den mündlichen Überlieferungen von Luhmanns Aussagen zur „Tyrell-Luhmann-Kontroverse“ kleinere Fußnoten Luhmanns. So erklärt Luhmann (2000, S. 25) in Organisation und Entscheidung, dass sich Anzeichen mehren, dass der Begriff der informalen Organisation und mit ihm der Gruppenbegriff durch eine Theorie der Interaktionssysteme ersetzt wird“. Dadurch könnte man den theoretischen, nicht „sehr entwicklungsfăhigen Gruppenbegriff durch die Theorie der Interaktion unter Anwesenden ersetzen“. Bei dieser nur angedeuteten Theorieentscheidung bleiben meines Erachtens etliche Fragen offen: Was würde man sowohI für die Interaktions- als auch für die Gruppensoziologie gewinnen, wenn man die Entscheidung zwischen Gruppen und Interaktionen nicht als ein Entweder-Oder behandelt, sondern versucht, gerade über die Differenz von Gruppe und Interaktion zu interessanten Beobachtungsschemata zu kommen? Weswegen hängt der von Luhmann später aufgegebene Informalitätsbegriff am Konzept der Gruppe? Könnte man ihn nicht auch mit dem Begriff von Face-to-Face-Interaktionen rekonstruieren? Lässt sich die einleuchtende Kritik am Konzept der Gruppe in Organisationen zu einer Kritik an Gruppen als soziales System generell abstrahieren?

2 Aufgrund der Ähnlichkeit dieser Elemente ist erklärlich, weswegen es bei vielen Gruppencoachings, Gruppensupervisionen oder gruppendynamischen Trainings fließende Übergänge zu Freundschaftsgruppen zu geben scheint. Die wissenschaftliche Untersuchung dieses Übergangsphänomens steht meines Wissens bisher noch aus. 
3 Hier gibt es sicherlich Ähnlichkeiten zu Interaktionen und Organisationen. Die sehr hohe Gehorsamkeitsbereitschaft in Milgrams Stromstoßexperiment lässt sich durch die freiwillige Teilnahme an den Experimenten und die Tendenz zur Vermeidung der Peinlichkeit bei einem vorzeitigen Abbruch erklären (Kühl 2005: 94 ff.)

4 Etwas irritierend in einer frühen Formulierung Luhmanns (1965, S. 176) ist, dass er behauptet, dass „Arbeitsgruppen“ in Organisationen „Gruppen im Vollsinne“ sind. Er schränkt das „Vollsinne“ aber gleich wieder ein, indem er sagt, dass sie ,über die Mitgliedschaft selbst nicht verfügen“ können“ und dadurch ,in der Möglichkeit, eigene Normen zu setzen und durchzusetzen", stark behindert sind.

5 Es wäre interessant, Grenzfälle zu untersuchen. Von den Arbeitskollektiven in DDR-Betrieben oder auch Cliquen in Organisationen in Entwicklungsländern weiß man, dass sich hier nicht nur eine Diffusität von Beziehungen zwischen den Mitgliedern ausbilden konnte, sondern diese auch unter anderem aufgrund fehlender Möglichkeiten, die Mitgliedschaft zu kündigen, eine hohe Autonomie gegenüber der Organisation ausbildeten.

\section{Literatur}

Allert, T. (1997). Zwei zu Drei: Soziologische Anmerkungen zur Liebe des Paares. In: System Familie, 10, 31-43.

Aubert, V. (1972). Interessenkonflikte und Wertkonflikt: Zwei Typen des Konflikts und der Konfliktlösung. In: W.L. Bühl (Hrsg.). Konflikt und Konfliktstrategie (S. 178-205.). München: Nymphenburger Verlagshandlung.

Becker, H. \& Useem R.H. (1942). Sociological Analysis of the Dyad. In: American Sociological Review, 7, S. 13-26.

Belardi, N. (2002). Supervísion. Grundlagen, Techniken, Perspektiven. München: Beck.

Binger, L. (1974). Kritisches Plädoyer für die Gruppe. In: Kursbuch H. 37/1974, S. 1-25.

Brown, R.(2000). Group Processes. Dynamics Within and Between Groups. 2. Aufl. Malden, MA; Oxford: Blackwell.

Buchinger, K. (1992). Ist Teamsupervision Organisationsberatung? Zur Professionalisierung von Selbstreflexion. In: R. Wimmer (Hrsg.). Organisationsberatung. Neue Wege und Konzepte (S. 151-169). Wiesbaden: Gabler.

Doob, L.W. (1970). Resolving Conflict in Africa. New Haven: Yale University Press.

Edding, C. (2005). Abschied von der Gruppe, so wie wir sie kannten und liebten? - Ein Rückblick und ein Ausblick. In: Gruppenpsychotherapie und Gruppendynamik, 41, S. 3-22.

Fatzer, G. (1999). Organisationen fragen Organisationen an Trends zur Vernetzung in Supervision und Organisationsentwicklung. In: Supervision, H. 36/1999, S. 17-34.

Feltham, C. \& Dryden, W. (2002). Grundregeln der Supervision. Weinheim; Basel: Belz Verlag.

Fichter, J.H. (1968). Grundbegriffe der Soziologie. Wien; New York: Springer.

Freund, J. (1976). Der Dritte in Simmels Soziologie. In: H. Böhringer \& K. Gründer (Hrsg.). Ästhetik und Soziologie um die Jahrhundertwende „Georg Simmel“ (S. 90-101). Frankfurt a.M.: Klostermann.

Goffman, E. (1971). Verhalten in sozialen Situationen. Strukturen und Regeln der Interaktion im öffentlichen Raum. Gütersloh: Bertelsmann Fachverlag.

Goffman, E. (1982). Die Verrücktheit des Platzes. In: E. Goffman (Hrsg.). Das Individuum im öffentlichen Austausch. Mikrostudien zur öffentlichen Ordnung (S. 434-503). Frankfurt a.M.: Suhrkamp.

Hirsch, M. (2004). Gedanken zum Schwinden der Attraktivität analytischer Gruppenpsychotherapie. In: Gruppenpsychotherapie und Gruppendynamik, 40, S. 164-178.

Hofstätter, P.R. (1957). Gruppendynamik. Die Kritik der Massenpsychologie. Hamburg: Rowohlt. 
Homans, G.C. (1950). The Human Group. New York: Harcourt, Brace.

Horn, K. (1969). Politische und methodologische Aspekte gruppendynamischer Verfahren. In: Das Argument, 10, S. 261-283.

Huber, A. \& Müller, G.F. (1998). Die Gruppe als Vermittlerin individueller Lernprozesse. In: E. Ardelt-Gattinger, H. Lechner \& W. Schlögl (Hrsg.). Gruppendynamik. Anspruch und Wirklichkeit der Arbeit in Gruppen. (S. 218-233). Göttingen: Verlag für Angewandte Psychologie.

Kaube, J. (2000). Die Nachwachen der Bürodiener. Einlaß in den Nachlaß und die aktenkundigen Angestellten im System: Niklas Luhmann über Organisation und Entscheidung. In: Frankfurter Allgemeine Zeitung, 17.2.2000, S. 62.

Kaube, J. (2004). Ein Dreier mit dem Soziologen. In: Frankfurter Allgemeine Zeitung, 26.11.2004.

Kaufmann, J.-C. (2004). „Der Morgen danach“. Wie eine Liebesgeschichte beginnt. Konstanz: UVK.

Kieserling, A. (1999). Kommunikation unter Anwesenden. Studien über Interaktionssysteme. Frankfurt a.M.: Suhrkamp.

Kieserling, A. (2002). Öffentlichkeit als Zensurmechanismus. München: unveröff. Ms.

König, R. (1983). Die analytisch-praktische Doppelbedeutung des Gruppentheorems. Ein Blick in die Hintergründe. In: F. Neidhardt (Hrsg.). Gruppensoziologie. Perspektiven und Materialien. Sonderheft 25 der Kölner Zeitschrift für Soziologie und Sozialpsychologie (S. 36-64). Opladen: WDV.

Kühl, S. (2005). Ganz normale Organisationen. Organisationssoziologische Interpretationen simulierter Brutalitäten. In: Zeitschrift für Soziologie, 34, S. 90-111.

Kühl, S. (2008). Coaching und Supervision. Zur personenorientierten Beratung in Organisationen. Wiesbaden. VS-Verlag.

Lell, J. \& Menne, F.W. (Hrsg.) (1976). Religiöse Gruppen. Düsseldorf: Vandenhoek \& Ruprecht.

Looss, W. (1991). Coaching für Manager - Problembewältigung unter vier Augen. Landsberg: Moderne Industrie.

Looss, W. (1997). Unter vier Augen. Coaching für Manager. 4. völlig überarb. Aufl. Landsberg am Lech: Moderne Industrie.

Luhmann, N. (1964). Funktionen und Folgen formaler Organisation. Berlin: Duncker \& Humblot.

Luhmann, N. (1965). Spontane Ordnungsbildung. In: M.F. Morstein (Hrsg.). Verwaltung. Eine einführende Darstellung (S. 163-183). Berlin: Duncker \& Humblot.

Luhmann, N. (1969). Einfache Sozialsysteme. Vorlesungsmanuskript Universität Bielefeld 1969/1970 unveröff. Ms.

Luhmann, N. (1972). Die Organisierbarkeit von Religionen und Kirchen. In: J. Wössner (Hrsg.). Religion im Umbruch. Soziologische Beiträge zur Situation von Religion und Kirche in der gegenwärtigen Gesellschaft (S. 245-285). Stuttgart: Ferdinand Enke.

Luhmann, N. (1975a). Interaktion, Organisation, Gesellschaft. In: N. Luhmann (Hrsg.). Soziologische Aufklärung 2. Aufsätze zur Theorie der Gesellschaft (S. 9-20). Opladen: WDV.

Luhmann, N. (1975b). Strukturauflösung durch Interaktion. Ein analytischer Bezugsrahmen. Bielefeld: unveröff. Ms.

Luhmann, N. (1988). Therapeutische Systeme. Fragen an Niklas Luhmann. In: F.B. Simon (Hrsg.). Lebende Systeme: Wirklichkeitskonstruktionen in der systemischen Therapie (S. 169-189). Frankfurt a.M.: Suhrkamp.

Luhmann, N. (1990). Sozialsystem Familie. In: N. Luhmann (Hrsg.). Soziologische Aufklärung 5. 3. Aufl. (S. 189-209). Opladen: WDV.

Luhmann, N. (2000). Organisation und Entscheidung. Opladen: WDV.

Mattke, D. (2006). Gruppensupervision als Ort beruflicher Rollenfindung und Professionalisierung von Gruppenpsychotherapeuten. In: Gruppenpsychotherapie und Gruppendynamik, 42, S. 23-38. 
Milgram, S. (1974). Obedience to Authority. An Experimental View. New York et al.: Harper \& Row.

Nau, E. (1983). Gruppendynamik in Deutschland - Ein Überblick. In: F. Neidhardt (Hrsg.). Gruppensoziologie. Perspektiven und Materialien. Sonderheft 25 der Kölner Zeitschrift für Soziologie und Sozialpsychologie (S. 126-143). Opladen: WDV.

Neidhardt, F. (1979). Das innere System sozialer Gruppen. In: Kölner Zeitschrift für Soziologie und Sozialpsychologie, 31, S. 639-660.

Neidhardt, F. (1983). Themen und Thesen zur Gruppensoziologie. In: F. Neidhardt (Hrsg.). Gruppensoziologie. Perspektiven und Materialien. Sonderheft 25 der Kölner Zeitschrift für Soziologie und Sozialpsychologie (S. 12-34). Opladen: WDV.

Oevermann, U. (2001). Strukturprobleme supervisorischer Praxis. Eine objektiv hermeneutische Sequenzanalyse zur Überprüfung der Professionalisierungstheorie. Frankfurt a.M.: Humanities Online.

Parsons, T. (1939). The Professions and Social Structure. In: Social Forces, 17, S. 457-467.

Rappe-Giesecke, K. (1990). Theorie und Praxis der Gruppen- und Teamsupervision. Berlin: Springer.

Richter, H.-E. (1972). Die Gruppe. Reinbek: Rowohlt.

Rühmkorf, P. (2004). Tabu II. Tagebücher 1971-1972. Reinbek: Rowohlt.

Schmidt, J.F.K. (2000). Die Differenzierung persönlicher Beziehungen: Das Verhältnis von Liebe, Freundschaft und Partnerschaft. In: K. Hahn \& G. Burkart (Hrsg.) (2000). Grenzen und Grenzüberschreitungen der Liebe. Studien zur Soziologie intimer Beziehungen II (S. 95-123). Opladen: Leske + Budrich.

Schreyögg, A. (1991). Organisationsanalytisches Wissen in der Supervision sozialer Arbeitsfelder. In: Gruppendynamik, 22, S. 5-18.

Schreyögg, A. (1992). Supervision. Ein integratives Modell. Lehrbuch zu Theorie und Praxis. 2. Aufl. Paderborn: Junfermann.

Simmel, G. (1992). Soziologie. Untersuchungen über die Formen der Vergesellschaftung. Frankfurt a.M.: Suhrkamp.

Slater, P.E. (1963). On Social Regression. In: American Sociological Review, 28, S. 339-364.

Slater, P.E. (1966). Microcosm. Structural, Psychological and Religious Evolution in Groups. New York; London; Sydney: Wiley \& Sons.

Stichweh, R. (1996). Professionen in einer funktional differenzierten Gesellschaft. In: A. Combe \& W. Helsper (Hrsg.). Pädagogische Professionalität. Untersuchungen zum Typus pädagogischen Handelns (S. 49-69). Frankfurt a.M.: Suhrkamp.

Tenbruck, F.H. (1972). Die moderne Gesellschaft. Freiburg, Basel, Wien: Herder.

Tyrell, H. (1983). Zwischen Interaktion und Organisation I: Gruppe als Systemtyp. In: F. Neidhardt (Hrsg.). Gruppensoziologie. Perspektiven und Materialien. Sonderheft 25 der Kölner Zeitschrift für Soziologie und Sozialpsychologie (S. 75-87). Opladen: WDV.

Uhlendorff; H. \& Oswald, H. (2003). Freundeskreise und Cliquen im frühen Jugendalter. In: Berliner Journal für Soziologie, 13, S. 197-212.

Utz, R. (1997). Soziologie der Intrige. Berlin: Duncker und Humblot.

Wetzel, J. (1998). Problemlösen in Gruppen: Miteinander ist besser als gegeneinander. In: E. Ardelt-Gattinger, H. Lechner \& W. Schlögl (Hrsg.). Gruppendynamik. Anspruch und Wirklichkeit der Arbeit in Gruppen (S. 113-126). Göttingen: Verlag für Angewandte Psychologie.

Whyte, W.F. (1943). The Street Corner Society. Chicago: The University of Chicago Press.

Wiese, L. von (1966). System der Allgemeinen Soziologie als Lehre von den sozialen Prozessen und den sozialen Gebilden der Menschen. 4. Aufl. Berlin: Duncker und Humblot.

Wiese, L. von \& Becker, H. (1932). Systematic Sociology. New York: Wiley. 
Zum Autor:

Stefan Kühl, Soziologe und Historiker, Professor für Soziologie an der Universität Bielefeld. Forschungsschwerpunkte: Gesellschaftstheorie, Organisationssoziologie, Interaktionssoziologie, Industrie- und Arbeitssoziologie, Professionssoziologie, Wissenschaftsgeschichte. 